\title{
Design of an Intelligent Combat Robot for war fields
}

\author{
Dr.S.Bhargavi \\ Electronics and Communication Engineering \\ S.J.C.I.T \\ Chikballapur, Karnataka, India
}

\author{
S.Manjunath \\ Electronics and Communication Engineering \\ S.J.C.I.T \\ Chikballapur, Karnataka, India
}

\begin{abstract}
The objective of this paper is to minimize human casualties in terrorist attack such as $26 / 11$. The combat robot [1] has been designed to tackle such a cruel terror attacks. This robot is radio operated, self- powered, and has all the controls like a normal car. A wireless camera has been installed on it, so that it can monitor enemy remotely when required. It can silently enter into enemy area and send us all the information through its' tiny Camera eyes. This spy robot can be used in star hotels, shopping malls, jewellary show rooms, etc where there can be threat from intruders or terrorists. Since human life is always precious, these robots are the replacement of fighters against terrorist in war areas.
\end{abstract}

Keywords-Combat Robot; Wireless camer; Terror attack; Radio Operated; Self-Powered; Intruders

\section{INTRODUCTION}

The global focus on terrorism and security may have geared up following the $9 / 11$ attacks in the USA. The risk of terrorist attack can perhaps never be eliminated, but sensible steps can be taken to reduce the risk. The word "Robot" was first used in a 1921 play titled R.U.R. Rossum's Universal Robots, by Czechoslovakian writer Karel Capek . Robot is a Czech word meaning "worker."

Merriam-Webster defines robot [2] as "a machine that looks like a human being and perform various complex acts; a device that automatically performs complicated, often repetitive tasks; a mechanism guided by automatic controls." ISO describes a robot as "an automatically controlled reprogrammable, multipurpose manipulator programmable in three or more axes, which may be either fixed in place or mobile for use in industrial automation applications".

Yet, all these definitions do give us a rough idea about what comprises a robot, which needs to sense the outside world and act accordingly. There are motors, pulleys, gears, gearbox, levers, chains, and many more mechanical systems, enabling locomotion. There are sound, light, magnetic field and other sensors that help the robot to collect information about its environment. There are Processors powered by powerful software that help the robot make sense environmental data captured and tell it what to do next and also microphones, speakers, displays, etc that help the robot interact with humans.

The main objectives of using robot are

A. Where man dares not venture
Robots have traditionally been put to use in environments that are too hazardous for man.

\section{B. $\quad$ To rescue, pronto!}

Robots also work under precarious conditions, for search and rescue after disasters. A host of robots built by the University of South Florida's Centre for robot assisted search and rescue were in action at the world trade centre site within hours after the disaster to delve into the rubble and rescue survivors. Similarly, robots are also put to work in underground mines. A lot of research today is focused on improving rescue functions of robots.

\section{We even make them go to war}

The faithful robots do not hesitate to tread even the dreaded terrain of battlefields [3]. Their use in Afghanistan and Iraq wars make us wonder if robots have indeed become intelligent! Battle robots of various shapes and sizes were deployed to defuse landmines, search for criminals hiding in caves, search for bombs under cars and in building, for espionage and what not! These robots were controlled by humans.

We aim to develop a model which will be efficiently used to minimize terrorist causality. Being able to achieve reliable long distance communication is an important open area of research to robotics as well as other technology areas. As interest in robotics continues to grow, robots are increasingly being integrated into everyday life. The results of this integration are end-users possessing less and less technical knowledge of the technologies [4].

Currently, the primary mode for robot communication uses RF. RF is an obvious choice for communication since it allows more information to be transferred at high speed and over long distance. This paper explores the use of readymade RF networks for communication and device control. This eliminates the need of a new infrastructure and detailed technical research.

\section{HARDWARE IMPLEMENTATION}

The block diagram of the hardware implementation of the entire system is as shown in the Figure 1. This robot is radio operated, self-powered and has all the controls like a normal car. A pair of laser gun has been installed on it, so that it can fire on enemy remotely when required. Wireless camera will send real time video and audio signals, which could be seen on a remote monitor, and action can be taken accordingly. 


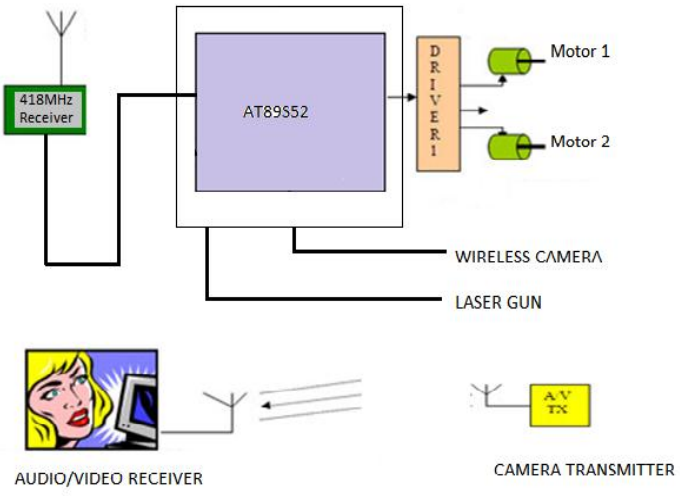

Figure 1: Block Diagram of Intelligent Combat Robot

Heart of our robot is Atmel's AT89S52 [5]. Microcontroller acts as master controller decodes all the commands received from the transmitter and give commands to slave microcontroller. It also acts as Slave microcontroller which is responsible for executing all the commands received from the master and also generating PWM pulses for the speed control. Based on the input codes master will give command to slave microcontroller and robot will behave as follows.

- moves in forward direction

- moves in reverse direction,

- $\quad$ speed controls in both the direction

- it can even turn left or right while moving forward or in reverse direction.

- Instant reverse or forward running without stopping.

\section{A. Transmitting unit}

Here a variable frequency oscillator 1 is used for modulating the frequency i.e. to be transmitted and has its output to a high frequency oscillator 2 for generating a carrier wave. The carrier wave is then radiated into space by the antenna. The transmitter module is shown in Figure 2.

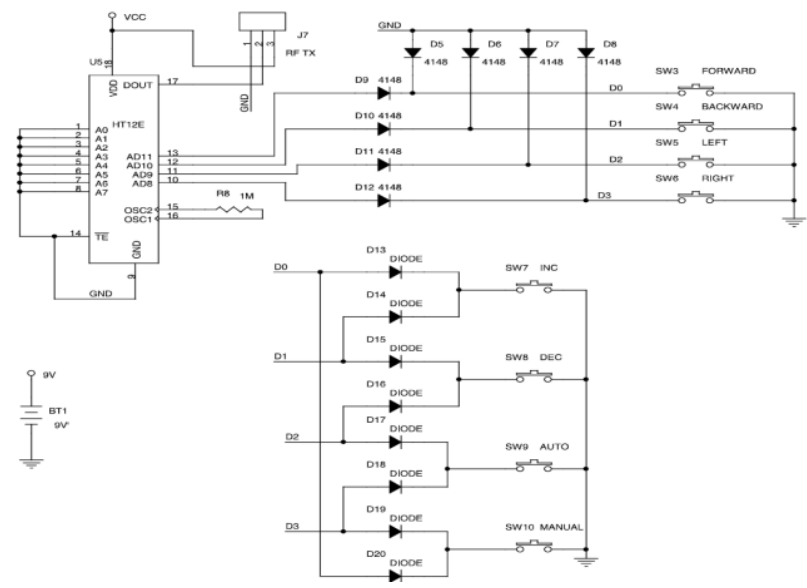

Figure 2: Transmitter Module

\section{B. Receiving Unit}

The receiving antenna is connected to a tuned wave detecting circuit for detecting the waves transmitted by transmitter antenna.The output of the tuned wave detecting circuit is connected to amplifier which in turn has its output connected to the input of the high pass frequency as well as the filter to a low pass frequency filter.

The outputs of amplifiers are connected to separate motors and other side of motors are connected to voltage potential .The high pass frequency filter extracts the higher frequency components of the output signals from the amplifier and the low pass frequency filter extracts the lower frequency components of the output signal from the amplifier .The receiver module is shown in Figure 3.

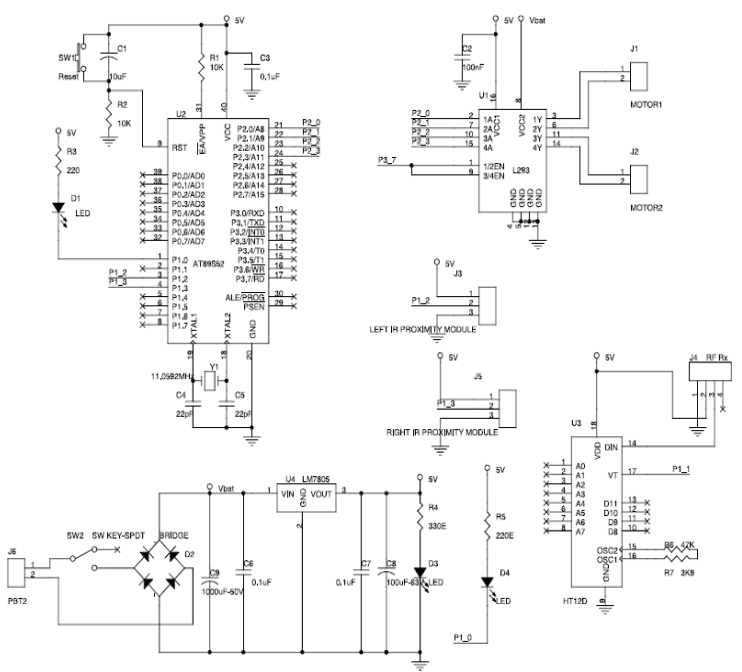

Figure 3: Receiver Module

\section{COMPONENTS OR SUBSYSTEMS DESCRIPTION}

\section{A. Microcontroller circuit (AT89S52)}

It is the heart of the system which controls all the activities of transmitting and receiving. The IC used is AT89S52. The AT89S52 Microcontroller [6] is an 8-bit microcontroller with $8 \mathrm{~K}$ Bytes of In-System Programming Flash Memory. The device is manufactured using Atmel's high-density nonvolatile memory technology and is compatible with the industry standard 80C51 instruction set and pin out. The on-chip Flash allows the program memory to be reprogrammed in-system or by a conventional nonvolatile memory programmer.

By combining a versatile 8-bit CPU with in-system programmable Flash on a monolithic chip, the Atmel AT89S52 is a powerful microcontroller which provides a highly-flexible and cost-effective solution to many embedded control applications. The Idle Mode stops the CPU while allowing the RAM, timer/counters, serial port, and interrupt system to continue functioning. The Power-down mode saves the RAM contents but freezes the oscillator, disabling all other chip functions until the next interrupt or hardware reset.

\section{B. Power supply circuit}

The main building block of any electronic system is the power supply to provide required power for their operation and is as shown in the Figure 4. For the microcontroller, keyboard, LCD, RTC, GSM, $+5 \mathrm{~V}$ are required \& for driving buzzer $+12 \mathrm{~V}$ is required. The power supply [7] provides regulated output of $+5 \mathrm{~V} \&$ non-regulated output of $+12 \mathrm{~V}$. The 
three terminals IC7805 meets the requirement of $+5 \mathrm{~V}$ regulated. The secondary voltage from the main transformer is rectified by electronic rectifier \& filtered by capacitor. This unregulated DC voltage is supplied to the input pin of regulator IC. The IC used are fixed regulator with internal short circuit current limiting \& thermal shutdown capability.

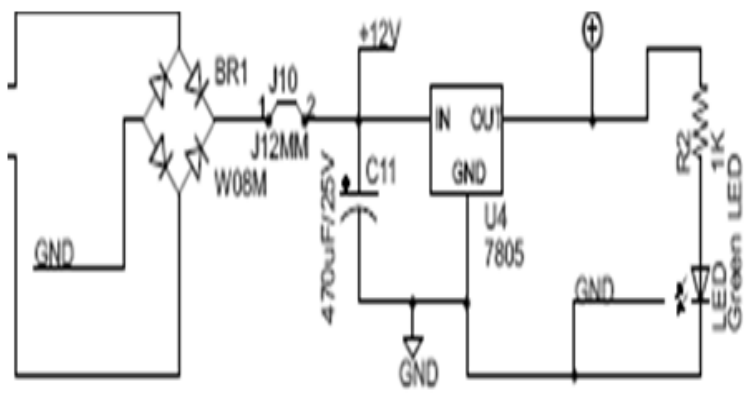

Figure 4: Power Supply Module

\section{Decoder HT-12D}

The decoders [8] are a series of CMOS LSIs for remote control system applications. They are paired with Holtek $2^{12}$ series of encoders. For proper operation, a pair of encoder/decoder with the same number of addresses and data format should be chosen.

The decoders receive serial addresses and data from a programmed $2^{12}$ series of encoders that are transmitted by a carrier using an RF or an IR transmission medium. They compare the serial input data three times continuously with their local addresses. If no error or unmatched codes are found, the input data codes are decoded and then transferred to the output pins.

The VT pin also goes high to indicate a valid transmission. The $2^{12}$ series of decoders are capable of decoding information's that consist of $\mathrm{N}$ bits of address and $12 \_\mathrm{N}$ bits of data. Of this series, the HT12D is arranged to provide 8 address bits and 4 data bits, and HT12F is used to decode 12 bits of address information

\section{Encoder HT-12E}

The $2^{12}$ encoders are a series of CMOS LSIs for remote control system applications. They are capable of encoding information which consists of $\mathrm{N}$ address bits and 12_N data bits. Each address/data input can be set to one of the two logic states. The programmed addresses/data are transmitted together with the header bits via an RF or an infrared transmission medium upon receipt of a trigger signal. The capability to select a TE trigger on the HT12E [8] further enhances the application flexibility of the $2^{12}$ series of encoders.

\section{E. DC Motors}

For the movement of our robot, we are using DC motors [9]. It is operated by $12 \mathrm{~V}$ DC power supply. In any electric motor, operation is based on simple electromagnetism. A current carrying conductor generates a magnetic field; when this is then placed in an external magnetic field, it will experience a force proportional to the current in the conductor, and to the strength of the external magnetic field.

\section{F. $\quad$ Motor Driver L293D}

The Device is a monolithic integrated high voltage, high current four channel driver designed to accept standard DTL or TTL logic levels and drive inductive loads and switching power transistors. To simplify use as two bridges each pair of channels is equipped with an enable input.

A separate supply input is provided for the logic, allowing operation at a lower voltage and internal clamp diodes are included. This device is suitable for use in switching applications at frequencies up to $5 \mathrm{kHz}$. The L293D is assembled in a 16 lead plastic package which has 4 center pins connected together and used for heat sinking. The chip is designed to control 2 DC motors. There are 2 Input and 2 output pins for each motor. The behavior of motor for various input is shown in Table 1.

TABLE 1. BEHAVIOR OF MOTORS

\begin{tabular}{|l|l|l|}
\hline Operation & A & B \\
\hline Stop & Low & Low \\
\hline Clockwise & Low & High \\
\hline Anti Clockwise & High & Low \\
\hline Stop & High & High \\
\hline
\end{tabular}

\section{G. Transmitter for Laser Gun}

The transmitter is constituted by AT90S2323 microcontroller and TLP434 RF transmitter module at $418 \mathrm{MHz}$. Transmitter is designed for more battery economy and safe transmission of the data.

Block diagram for the transmitter of laser gun is as shown in the Figure 5.

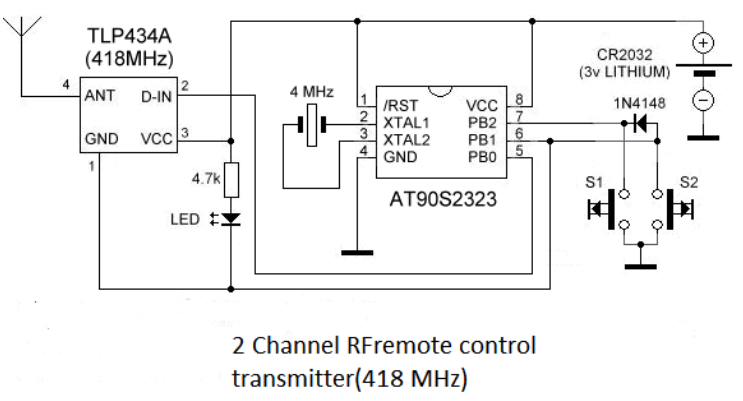

Figure 5: Block Diagram of Transmitter Laser Gun

Here TLP434A is an Ultra-small Trasmitter of range $418 \mathrm{MHz}$ with ASK modulation scheme with operating voltage range from 2-12 dc voltage. This IC is usually chained with the encoder IC for example HT12-E. This transmitter is connected to the AT90S2323 10MHz with $2 \mathrm{k}$ flash 
microcontroller. This constitutes a transmitter section of laser gun.

\section{H. Receiver for Laser Gun}

The receiver constituted by $\mathrm{RF}$ receiver module RLP434A at 418MHz, the microcontroller AT90S2313 [10] and the two relays which can handle any electric (or electronic) device up to 10 Amps (the contacts of my relays are $10 \mathrm{Amp}$ at 250 Volts). The RLP434A is an RF receiver module with receipt frequency at $418 \mathrm{MHz}$ with ASK modulation. There are 2 outputs from this module, the digital, with levels from 0v to VCC (5 volts in our case) and the analog output. Analog output is not used. The transmitter sends 4 bytes with 2400bps 4 times and the receiver RLP-434A collects them and moves them to AT90S2313 to $\mathrm{RxD}$ pin, PD0. Block diagram for receiver of the laser gun is depicted in Figure 6.

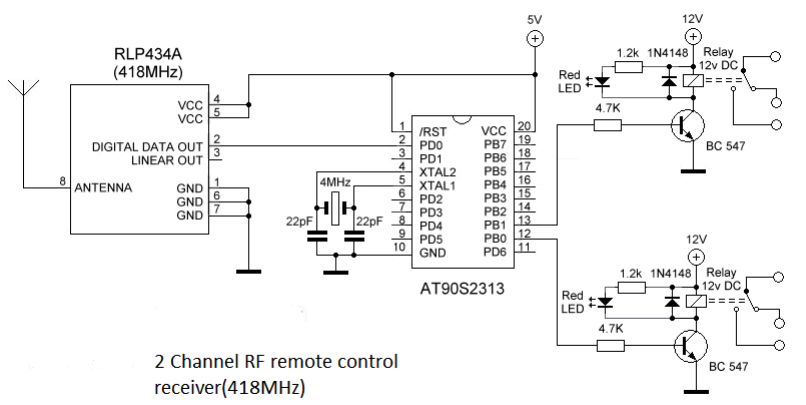

Figure 6: Block Diagram of Receiver Laser Gun

Here RL434A is SAW (surface acoustic wave) based receiver compatible of $418 \mathrm{MHz}$ of frequency with operating range from 3.3-6 dc voltage and also it employs ASK modulation. Again as a Tx, here also we have 8-bit microcontroller AT90S2313 with 2k flash memory with 11 pin DIP . All these components will constitute a receiver of laser gun.

\section{RF Communication}

Radio frequency (RF) is a rate of oscillation in the range of about $3 \mathrm{kHz}$ to $300 \mathrm{GHz}$, which corresponds to the frequency of radio waves, and the alternating currents which carry radio signals. RF usually refers to electrical rather than mechanical oscillations. The energy in an RF current can radiate off a conductor into space as electromagnetic waves (radio waves); this is the basis of radio technology.

\section{J. JMK AV Receiver with Wireless Camera}

It is mini wireless monitoring video camera and wireless receiver set for home and small business surveillance and is used here for demonstration purpose. Simply install the wireless camera in the room where we want to monitor and set the wireless receiver in the next room (up to 15 meters away) and hook it up to a TV or DVR to watch the action or record the footage for the security records.

Here we are placing this wireless camera in the combat robot. Depiction of AV Receiver wireless camera is as shown in Figure 7.

\section{K. TV Capture card}

A TV capture card is a computer component that allows television signals to be received by a computer. It is a kind of television tuner. Most TV tuners also function as video capture cards, allowing them to record television programs onto a hard disk. Digital TV tuner card is as shown in the Figure 8.

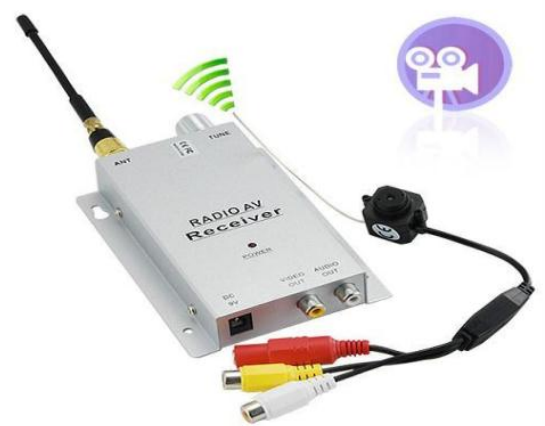

Figure 7: AV Receiver and Wireless Camera

The card contains a tuner and an analog-to-digital converter along with demodulation and interface logic.

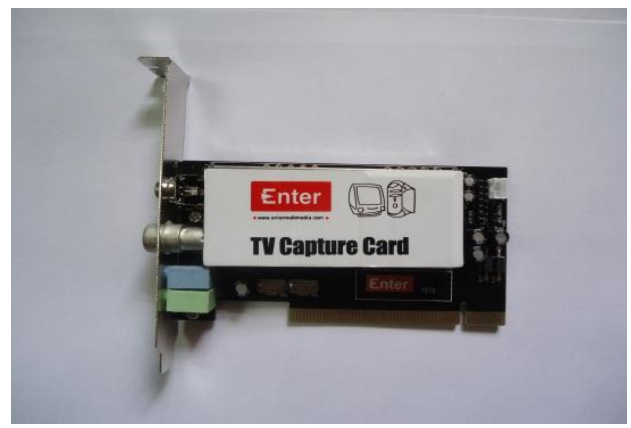

Figure 8: ATI digital TV capture card

\section{L. $\quad$ Remote Controller Decoder SC2272-T4}

It can be used for wireless remote control receivers and its features are

- Operating voltage: DC 4 6V.

- Up to 12 tri-state code address pins.

- Up to 6 data pins.

- Toggle control mode.

\section{SOFTWARE IMPLEMENTATION}

For the software implementation, we deploy two software packages. First one is the Keil $\mu$ Vision 3.0. second one is the Flash magic simulator.The Keil $\mu$ Vision Debugger accurately simulates on-chip peripherals $\left(\mathrm{I}^{2} \mathrm{C}, \mathrm{CAN}, \mathrm{UART}\right.$, SPI, Interrupts, I/O Ports, A/D Converter, D/A Converter, and PWM Modules) of 89S52 device.

Simulation helps to understand hardware configurations and avoids time wasted on setup problems. With simulation, we can write and test applications before target hardware is available. The system program written in embedded C [11] using KEIL IDE software will be stored in Microcontroller.

Keil development tools for the Microcontroller Architecture support every level of software developer from 
the professional applications engineer to the student for learning about embedded software development. The industry-standard Keil C Compilers, Macro Assemblers, Debuggers, Real-time Kernels, Single-board Computers, and Emulators support all 89S52 derivatives. The Keil Development Tools are designed to solve the complex problems facing embedded software developers.

Flash magic is used to dump the code to microcontroller from PC. Flash Magic is a free, powerful, feature-rich Windows application that allows easy programming of Philips FLASH Microcontrollers. Build custom applications for Philips Microcontrollers on the Flash Magic platform! Use it to create custom end-user firmware programming applications, or generate an in-house production line programming tool.

The Flash Memory In-System Programmer is a tool that runs under Windows 95/98/NT4/2K. It allows in-circuit programming of FLASH memories via a serial RS232 link. Computer side software called Flash Magic is executed that accepts the Intel HEX format file generated from compiler Keil to be sent to target microcontroller. It detects the hardware connected to the serial port.

\section{A. Flow charts}

The flowcharts depicting the Robot Movement and its Delay are shown in Figure9, 10 and 11.

\section{1) Robot Movement}

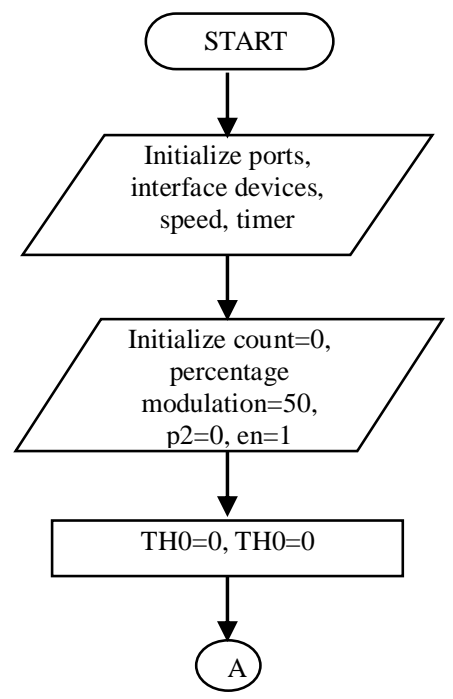

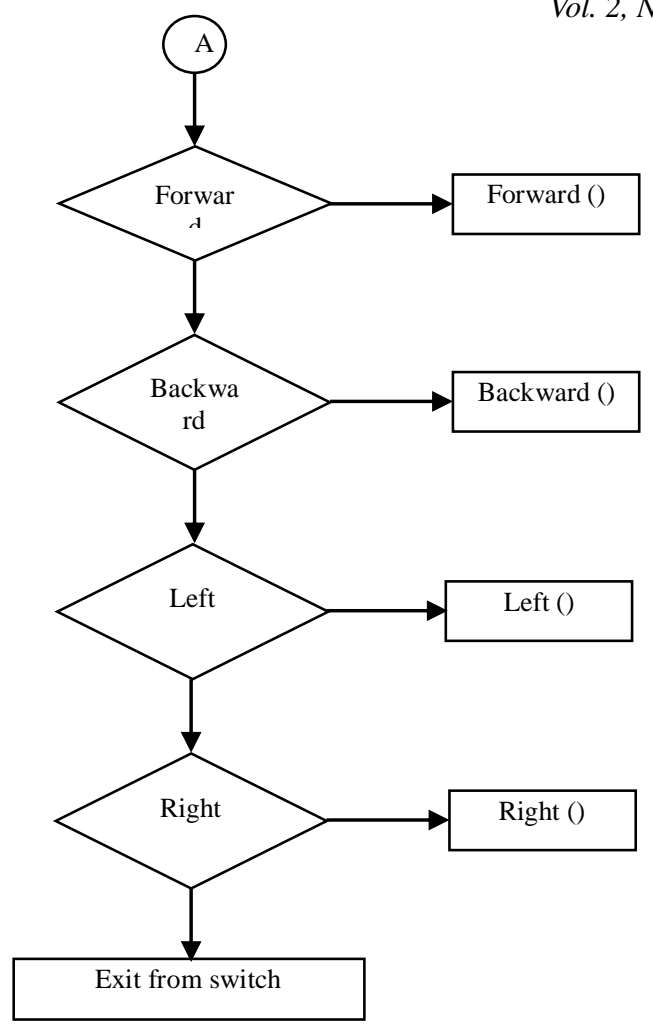

Figure 9: Flowchart For Robot Movement

2) Robot for Particular Movement

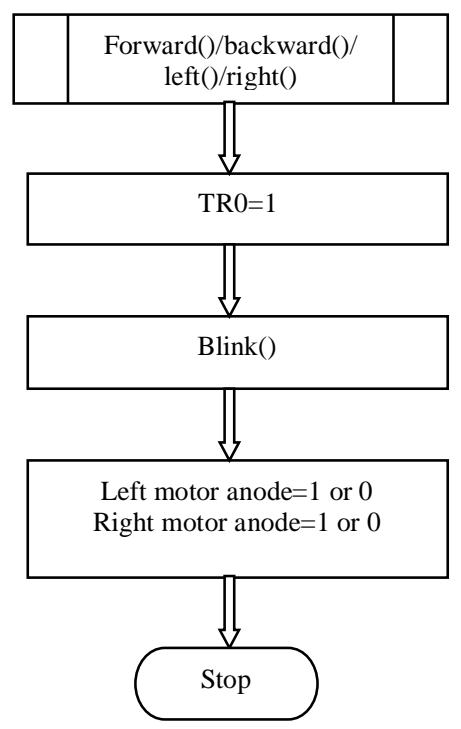

Figure 10: Flowchart For Particular Movement 


\section{3) Delay Flowchart}

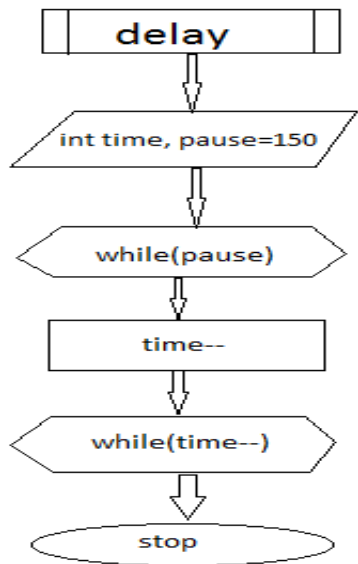

Figure 11: Flowchart For Robot Movement

\section{RESULTS AND DISCUSSION}

Remote controllers are designed to direct the orientation of robot and to operate the laser gun. Robot keeps on moving in two modes i.e., Manual mode and self-mode. It's brought under user's control in the case of manual mode. In self-mode, robot starts moving over surface and takes action according to the scenario. To detect the obstacles, we have deployed Infrared sensors (left sensor and right sensor) in the front portion of the module. While moving on the surface, if the left sensor is detected, robot takes back the position for a moment and moves right. If the right sensor is detected, robot gets back and moves left. The front view and top view of designed combat robots are shown in the figures $12 \& 13$.

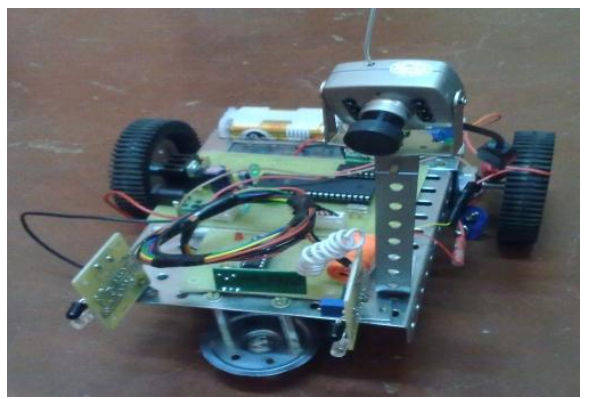

Figure 12: Front view of designed combat robot

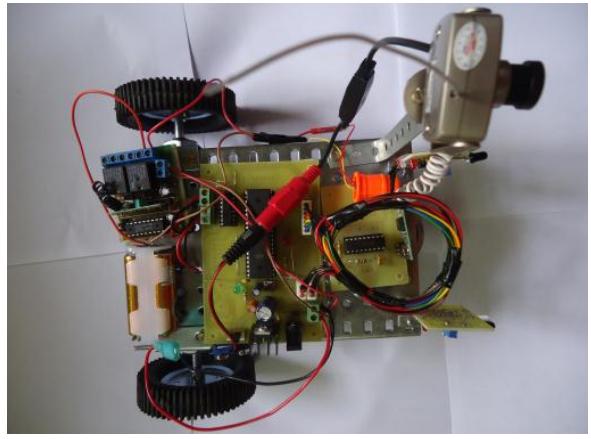

Figure 13: Top view of designed combat robot

\section{APPLICATIONS}

- Can be adequately implemented in national defense through military-industrial partnership. It is shown in the figure 14.

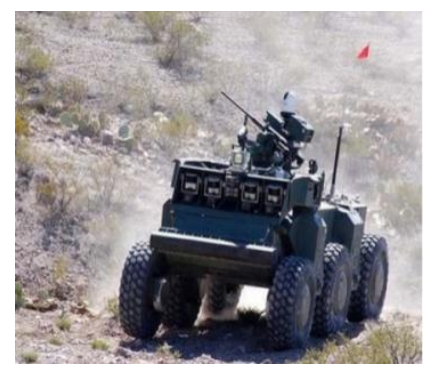

Figure 14: Top view of combat robot

- Can be vastly applied in Resorts, borders of noted buildings. It is shown in the figure 15 .

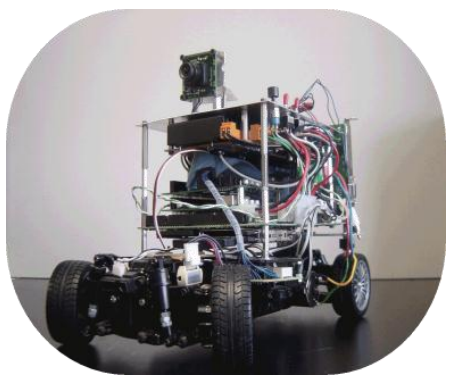

Figure 15: Top view of combat robot

- Installation of combat robots in the stadiums, sacred places, government and non government organizations assures top security.

\section{CONCLUSION}

As we all know, these days India is sick off massive terror attacks, bomb explosions at plush resorts. To avoid such disasters TECHNOLOGICAL power must exceed HUMAN power. Human life and time are priceless.

It's our onus to take an initiative to design a model of an apt robot that meets combatant needs. So to avoid terror attacks, to ensure more security at the border and high density areas it's wise to maintain a world class military technology in accordance with combatant needs.

Even every nation needs its own defense system for their integrity and security. In such a way construction of these robots will carry nation's name, fame globally.

\section{REFERENCES}

[1] Pete Miles \& Tom Carroll, Build Your Own Combat Robot, (2002).

[2] K.S.Fu, R.C.Gonzalez, C.S.G..Lee, Tutorials Robotics.

[3] Asaro,P. How just could a robot war be?, Frontiers in Artificial Intelligence and Applications, 75, 50-64.

[4] S. Y. Harmon \& D. W. Gage, "Current Technical Research Issues of Autonomous Robots Employed In Combat", 17th Annual Electronics and Aerospace Conference. 
[5] www.Atmel.com

[6] Atmel data

http://www.keil.com/dd/docs/datashts/atmel/at89s52_ds.pdf

[7] Robert L.Boylestad and Louis Nashelsky, "Electronic Devices and Circuit Theory", 8th Edition, 2006 [8] Decoder $\quad$ HT-12D,
http://robokits.co.in/shop/index.php?main_page=product_info\& cPath $=14 \_15 \&$ products_id=76>

[9] A. Khamis, M. Pérez Vernet, K. Schilling, "A Remote Experiment On Motor Control Of Mobile Robots", 10thMediterranean Conference on Control and Automation - MED2002.

[10] Receiver for

Laser

Gun www.alldatasheet.com/datasheet-pdf/pdf/169605/...RLP434A.htm

\section{AUTHORS PROFILE}

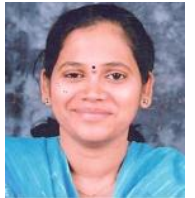

Dr.S.Bhargavi is presently working as a Professor in the department of Electronics and Communication engineering, SJCIT, Chikballapur, Karnataka, India. She is having 12 years of teaching experience. Her areas of interest are Robotics, Embedded Systems, Low Power VLSI, Wireless communication, ASIC and Cryptography.

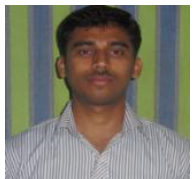

S.Manjunath is presently working as a Lecturer in the department of Electronics and Communication engineering, SJCIT, Chikballapur, Karnataka, India. He is having 3 years of Industrial experience and 1 year of teaching experience. His areas of interest are Robotics, Embedded Systems and Wireless communication. 